\title{
USE OF LABORATORY AND IMAGING
INVESTIGATIONS IN DEMENTIA
}

W M van der Flier, P Scheltens

J Neurol Neurosurg Psychiatry 2005; 76(Suppl V):v45-v52. doi: 10.1136/jnnp.2005.082149

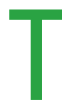

he diagnosis of dementia is a complicated enterprise. This is partly caused by the insidious onset of most diseases that cause dementia. The difficulty is clearly captured in the question:

"Where does ageing stop and dementia begin?". The availability of symptomatic treatment (for example, acetyl cholinesterase inhibitors) and the development of new disease modifying drugs emphasises the need for improved diagnostic accuracy. Patients that are in the earliest stages of the disease may potentially profit most from disease modifying drugs. This further underlines the importance of a correct clinical diagnosis early in the course of the disease. During lifetime, with careful evaluation and the application of well defined, clinical criteria, "probable" diagnoses can be made with a relatively high level of accuracy. A definite diagnosis of Alzheimer's disease $(A D)$ or most other diseases that cause dementia can only be made post mortem, after necropsy.

Neuropathology accumulates gradually, and it has been shown that neuropathological features, eventually leading to the clinical syndrome of dementia, may be present as early as 20 years before the first symptoms become overt. Therefore, based on clinical criteria alone, the diagnosis cannot be made until the disease is in a relatively far advanced stage. There is a clear need for sensitive and reliable biologic markers that are able to demonstrate the presence of neuropathology before a patient has reached the stage of clinical dementia. Although as yet there are no biomarkers that can diagnose $\mathrm{AD}$ or most other dementias with certainty, neuroimaging and laboratory markers may add positive evidence for the presence of neuropathology.

In this article, an overview will be given of the possibilities of diagnosing dementia. The first part consists of a short description of the general diagnostic work-up of dementia. Due to the general character of this overview, we will not go into the clinical criteria of the specific diseases that cause dementia. Rather, we will describe the general criteria for dementia as described in the Diagnostic and statistical manual of mental disorders, 4th ed (DSM IV). ${ }^{1}$ The second part will be dedicated to the quest for neuropathology underlying dementia, with use of laboratory and neuroimaging investigations. Some of these investigations, like magnetic resonance imaging (MRI), are already widely used in clinical practice, whereas others-for example, biomarkers obtained with lumbar puncture-hold promise for the future.

\section{CLINICAL CRITERIA}

The practice parameter of the American Academy of Neurology recommends as a guideline that the definition of dementia according to the DSM IV (which is identical to the DSM-III-R) is reliable, and should be used in the diagnostic work-up of dementia. ${ }^{12}$ According to the DSM IV, dementia is present if an individual develops multiple cognitive deficits that cause impairment in social or occupational functioning and represent a significant decline from a previous level of functioning (table 1).

A deterioration of cognitive function is central to the criteria of dementia. The diagnostic workup of dementia should therefore always begin by careful history taking in interviews with both the patient and a reliable informant. Can the patient and the informant describe the character, development, and progression of the cognitive complaints? Can they give examples? In this respect, it is important to remember that a cognitive complaint is not by definition the same as a

See end of article for authors' affiliations

Correspondence to: W M van der Flier, Department of Neurology and Alzheimer Center, Vriie Universiteit Medical Center, PO Box 7057, $1007 \mathrm{MB}$ Amsterdam, The Netherlands: wm.vdflier@vumc.nl cognitive deficit. For example, when a patient describes his or her memory problems in detail (an appointment $\mathrm{X}$ with person $\mathrm{Y}$ was forgotten on date $\mathrm{Z}$ ), this provides a clue that the memory for the problems may still be good enough. Although the patient presents with a complaint, this does not necessarily reflect a formal deficit. In a cohort of 1000 consecutive patients referred to a memory clinic because of cognitive complaints, $29 \%$ of patients had no demonstrable cognitive deficits. ${ }^{3}$ Dementia was diagnosed in $43 \%$. Finally, $28 \%$ of patients suffered isolated amnesia, other or uncertain cognitive deficits, or could not be classified.

The concept of mild cognitive impairment (MCI) refers to the transitional phase between normal and dementia. ${ }^{4}$ Typically, patients suffer from an isolated memory impairment, with global cognitive function still relatively intact (amnestic MCI). The concept of MCI has been 
Table 1 DSM IV criteria for dementia

(A) Development of multiple cognitive deficits manifested by both:

1. Memory impairment (impaired ability to learn new information or to recall previously learned information)

2. One (or more) of the following cognitive disturbances:

- aphasia (language disturbances)

apraxia (impaired ability to carry out motor activities despite intact motor function)

- agnosia (failure to recognise or identify objects despite intact sensory function)

- disturbance in executive functioning (that is, planning, organising, sequencing, abstracting

(B) Cognitive deficits in criteria $\mathrm{A} 1$ and $\mathrm{A} 2$ each cause significant impairment in social or occupational functioning and represent a significant decline from a previous level of functioning

(C) Deficits do not occur solely during a delirium

(D) Deficits not due to psychiatric disease (major depression, schizophrenia)

broadened to include patients with isolated impairment of another cognitive function than memory (non-amnestic MCI), and with mild impairment of multiple cognitive functions (multi-domain MCI). Patients with MCI are at an increased risk of developing dementia. Approximately 15\% of MCI patients progress to dementia (mostly AD) per year, as compared with $1-2 \%$ of the normal population. Although at an increased risk, not all patients with MCI develop dementia. Some MCI patients suffer incipient AD, whereas others have a benign form of cognitive deficits that will not progress.

In $\mathrm{AD}$, memory deficits are often the first and most salient symptoms. However, occasionally, AD may also present with agnosia, apraxia and aphasia being more prominent than memory deficits. Moreover, other types of dementia typically present with other profiles of cognitive dysfunction. For example, vascular dementia $(\mathrm{VaD})$ is characterised by mental slowness and executive dysfunctioning. Patients with frontotemporal lobar degeneration (FTLD) suffer primarily from executive dysfunctioning and/or aphasia. Moreover, behavioural problems may precede cognitive deficits by years. In addition to the interview with the patient and informant, observation of a patient is of the utmost importance. Is the patient able to relate his problems in a coherent way? If not, this may suggest aphasia. Does the patient behave in a "clumsy" manner when asked to put on reading glasses, or when asked to undress for the physical examination? Clumsiness may be a sign of apraxia. To get a first impression of general cognitive functioning, testing of mental status may prove valuable. Simple questions and tasks such as those of the mini mental state examination (MMSE) provide insight into the level of cognitive functioning of a patient (fig 1). The MMSE has a maximum score of 30. Scores below 25 are considered as congruent with a diagnosis of dementia. With a high level of education, a higher score may already be suggestive of cognitive dysfunction, while a score below 25 is by no means synonymous with dementia. The clock drawing test, in which a patient is asked to draw a clock and set the time for 10 after 11 can give an impression of visuospatial and executive functioning (fig 2). If the history and cognitive screening tests do not establish the diagnosis, referral to a neuropsychologist to provide a more detailed evaluation of memory and other cognitive functions is appropriate.

The standard diagnostic work-up of dementia should also include a general neurological and physical examination,
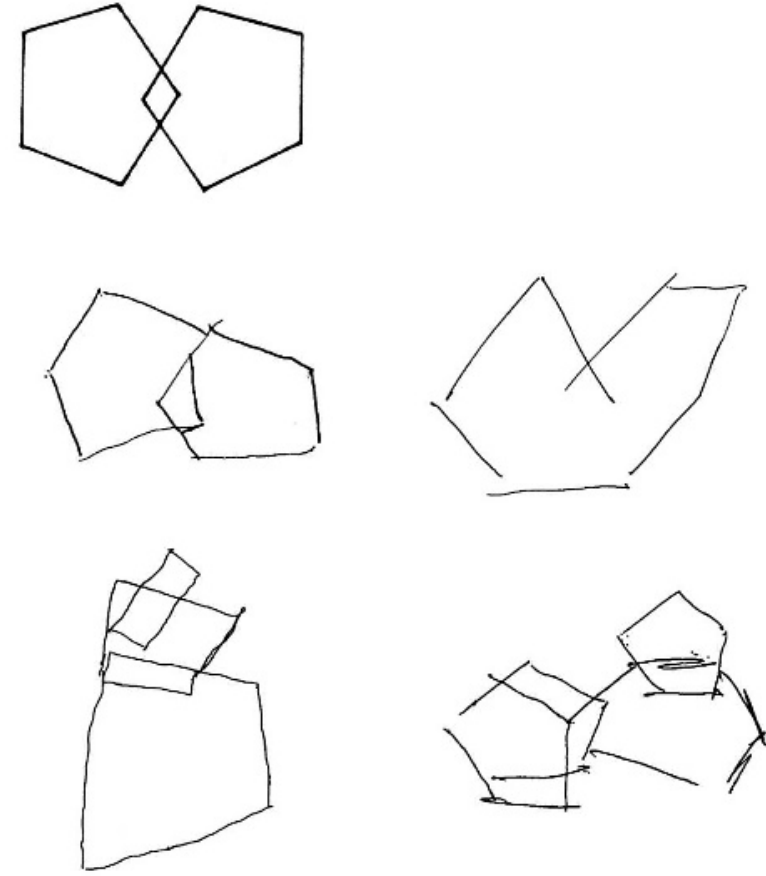

Figure 1 Example and copies of the interlocking pentagons from the mini mental state examination by patients with Alzheimer's disease (AD).
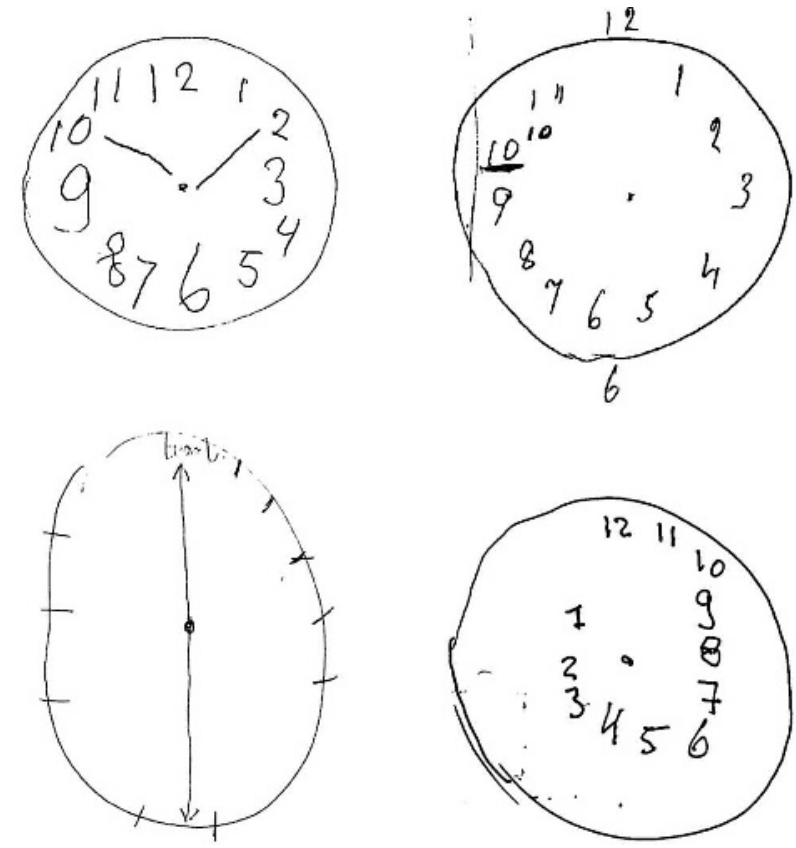

Figure 2 Clock drawings by patients with $A D$. In the clock drawing test, the patient is asked to draw a clock and set the time for 10 after 11 .

assessment of behavioural disturbances, and laboratory screening. The aims of laboratory investigations include (1) to identify co-morbidity and/or complications; (2) to reveal potential risk factors; (3) to explore the background of frequently associated confusional states; and (4) more rarely to identify the primary cause of dementia. The following blood tests are generally proposed for all patients at first evaluation: blood sedimentation rate; complete blood cell count; electrolytes; glucose; renal and liver function tests, 
thyroid stimulating hormone, serological test for syphilis, detection of B12. Assessment of co-morbidities is essential for the diagnosis and management of dementia. Treatable comorbidities that are commonly observed in the elderly, and in patients with suspected dementia in particular, include depression, B12 deficiency, and hypothyroidism. ${ }^{2}$ Although treatment of these disorders may not completely reverse cognitive dysfunction, they should be recognised and treated.

Based on clinical criteria alone, the diagnosis of dementia cannot be made until a relatively far advanced disease stage, as gradually accumulating neuropathology may be present many years before symptoms become clinically overt. With continuing attention of the media and public awareness for the growing public health problem resulting from dementia, patients tend to seek help in ever earlier stages. This poses the physician a problem, as clinical criteria are lacking in sensitivity in the earliest stages of disease. In response to the demand for reliable prognoses in the earliest stages of disease, diagnostic procedures turn from an exclusionary to an inclusionary approach. Traditionally, additional investigations were performed to rule out other potential causes of cognitive decline. Recent developments are resulting in the use of laboratory and neuroimaging investigations to seek positive evidence for the diagnosis of $\mathrm{AD}$ or other forms of dementia. Modern biomarkers aim to provide more direct evidence for the presence or absence of neuropathology, enabling meaningful prognoses and possible intervention in early disease stages. The following is an overview of currently used laboratory and imaging techniques. Some of these are already used in the standard diagnostic work-up of dementia, while others are still in an experimental stage.

\section{MAGNETIC RESONANCE IMAGING}

In the recent practice parameter on the diagnosis of dementia, structural neuroimaging in the routine initial evaluation of patients with dementia is recommended as a guideline. ${ }^{2}$ The traditional view has been that computed

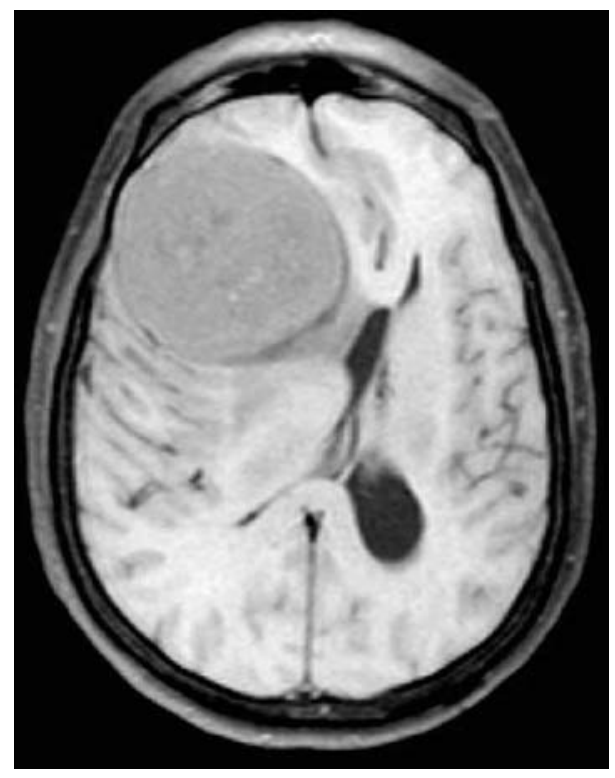

Figure 3 Axial magnetic resonance imaging (MRI) scan of a 60 year old woman with slowly progressive behavioural changes and memory deficits. The scan shows a large space occupying lesion in the right frontal lobe. The pathological diagnosis was meningeoma. tomography and magnetic resonance imaging (MRI) are performed to exclude other abnormalities that are potentially amenable to surgical treatment, such as a tumour (fig 3), haematoma, or hydrocephalus. In a memory clinic setting, such potentially reversible conditions detected by imaging appear to underlie about $4 \%$ of dementias. ${ }^{3}$ In addition to exclusion of gross structural pathology, imaging is increasingly being used to add positive or negative predictive value in the diagnosis of the more common dementing illnesses. ${ }^{5}$

\section{Medial temporal lobe}

Neuropathological studies have implicated the medial temporal lobe-including the hippocampus, amygdala, parahippocampal gyrus, and entorhinal cortex-as an early site of pathological involvement in $\mathrm{AD}$, and consequently many imaging studies have focused on this part of the brain. ${ }^{6}$ Hippocampal volume loss on MRI has been shown to predict subsequent fulfilment of neuropathological criteria of $\mathrm{AD}{ }^{7}$

Hippocampal volumes have been shown to be reduced in AD patients when compared to controls (fig 4). ${ }^{8}$ However, this finding is not specific for $\mathrm{AD}$, as hippocampal atrophy also occurs in other common dementias such as VaD, dementia with Lewy bodies (DLB), and FTLD. It is an unresolved issue as to whether different types of neuropathology - associated with the different types of dementiaresult in the same macroscopic feature of hippocampal atrophy, or if the hippocampal volume loss observed in different types of dementia reflects an overlap of neuropathology between dementias. Hippocampal atrophy is a relatively early marker of pathology. In patients with MCI, it has been shown to be predictive of progression to dementia, mostly AD.' Moreover, hippocampal volume loss has been observed in asymptomatic subjects at risk of autosomal dominant familial $\mathrm{AD} .^{10}$

Volumetric studies have shown that structures in the medial temporal lobe are specifically involved in neurodegenerative disease. However, due to the time intensive nature of the measurement, volumetry of the hippocampus is not easily employed in routine clinical practice. Visual rating scales, although cruder estimates of atrophy, can provide valuable tools that are more easily applicable in clinical practice. A widely used five point visual rating scale evaluates the width of the choroid fissure, the width of the temporal horn, and the height of the hippocampus (table 2). ${ }^{11}$ Sensitivity and specificity for detection of mild to moderate $\mathrm{AD}$ compared with controls based on the presence of atrophy of the medial temporal lobe is $85 \%$ and $88 \%$, respectively. ${ }^{5}$

\section{Specific pattern of atrophy}

Atrophy of the medial temporal lobe is suggestive of $\mathrm{AD}$, although it does not rule out other causes of dementia. A minority of patients with $\mathrm{AD}$ presents with prominent posterior cortical atrophy on MRI. These patients are also referred to as biparietal $\mathrm{AD}$. Other specific patterns of atrophy lower the probability of a diagnosis of AD. Frontal and temporal atrophy have been reported as suggestive of frontotemporal dementia (FTD), although absence of any abnormality is not uncommon in this disorder (fig 5). ${ }^{12}$ Asymmetrical, predominantly left sided atrophy can be observed in progressive non-fluent aphasia and semantic dementia. Progressive non-fluent aphasia typically presents with left sided perisylvian atrophy, whereas semantic dementia is characterised by left sided anterior temporal lobe atrophy. 


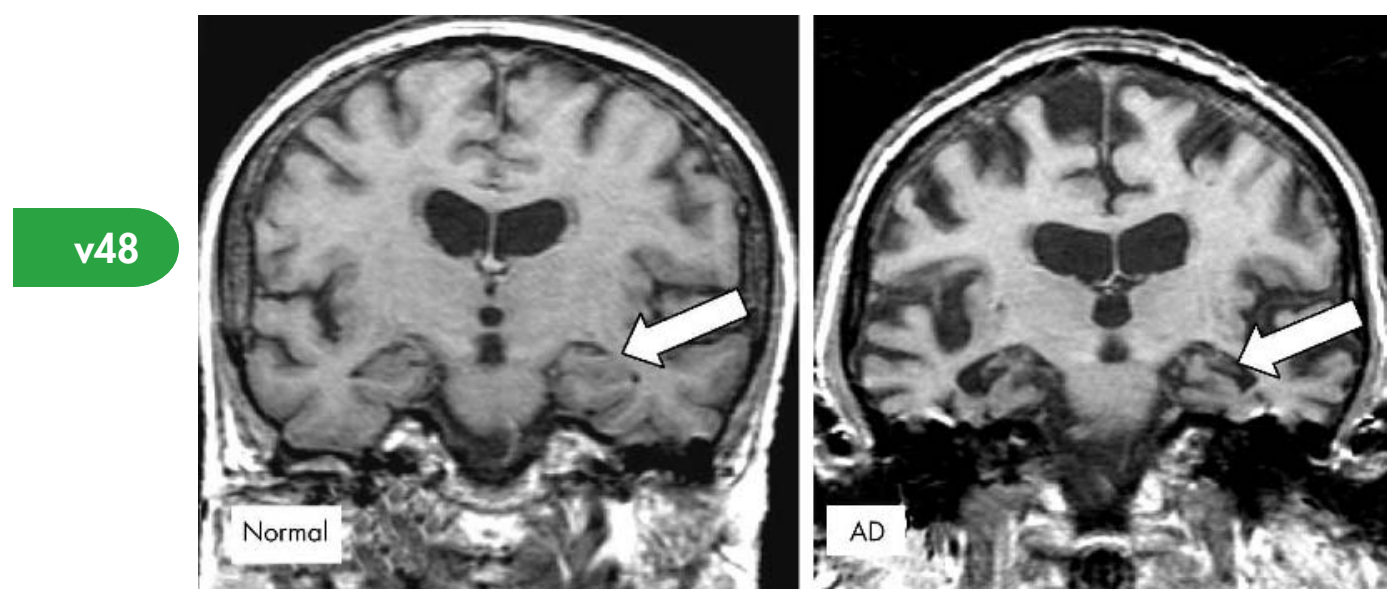

Figure 4 Coronal MRI scan shows absence (left) and presence (right) of atrophy of the medial temporal lobe in a healthy elderly subject and a patient with $A D$.

\section{Vascular pathology}

Imaging findings of vascular pathology include "large vessel" (large territorial or strategical infarctions) and "small vessel" (lacunes and white matter hyperintensities) disease. Criteria for VaD include definitions of dementia, vascular disease, and a relationship between the two. Vascular disease can be diagnosed on clinical grounds, neuroimaging, or both. The criteria according to the National Institute of Neurological Disorders and Stroke-Association Internationale pour la Recherche et l'Enseignement en Neurosciences (NINDSAIREN) specify clinical and radiological criteria of $\mathrm{VaD} .^{13}$ The radiological criteria require that the vascular territories involved are considered relevant for VaD. These include large vessel strokes in the areas of the anterior cerebral artery, posterior cerebral artery, association areas and watershed regions, and small vessel disease. However, considerable interobserver variability exists for the assessment of the radiological component of the NINDS-AIREN criteria. The use of operational definitions improves agreement, but only among experienced observers. ${ }^{14}$

Vascular disease is not only observed on MRI in patients with $\mathrm{VaD}$, but also in patients with $\mathrm{AD}$. In fact, many pathological studies find concomitant cerebrovascular disease in patients with definite AD. ${ }^{15}{ }^{16}$ Additional vascular pathology significantly increases the likelihood of clinical dementia, suggesting a synergistic effect. In addition, evidence of cerebrovascular pathology may influence the clinical management of the patient with dementia, given that cerebrovascular disease may be amenable to targeted interventions potentially ameliorating disease progression.

\section{POSITRON EMISSION TOMOGRAPHY}

It is conceivable that changes in brain function precede structural brain changes. The two main techniques that are

Table 2 Visual assessment of medial temporal lobe atrophy

\begin{tabular}{llll}
\hline Score & $\begin{array}{l}\text { Width of choroid } \\
\text { fissure }\end{array}$ & $\begin{array}{l}\text { Width of temporal } \\
\text { horn }\end{array}$ & $\begin{array}{l}\text { Height of } \\
\text { hippocampus }\end{array}$ \\
\hline 0 & Normal & Normal & Normal \\
1 & $\uparrow$ & Normal & Normal \\
2 & $\uparrow \uparrow$ & $\uparrow$ & $\downarrow \downarrow$ \\
3 & $\uparrow \uparrow \uparrow$ & $\uparrow \uparrow$ & $\downarrow \downarrow$ \\
4 & $\uparrow \uparrow \uparrow$ & $\uparrow \uparrow \uparrow$ & $\downarrow \downarrow \downarrow$ \\
\hline
\end{tabular}

$\uparrow$ increased; $\downarrow$ decreased. Reproduced with permission of BMJ Publishing Group. ${ }^{11}$ used to study biochemical and physiological processes are positron emission tomography (PET) and single photon emission computed tomography ( SPECT). PET shows glucose metabolism (using the metabolic tracer [18F]fluorodeoxyglucose or FDG). SPECT assesses changes in regional blood flow and oxygen metabolism. Both methods have reasonable to good discriminatory power in the comparison between $\mathrm{AD}$ and controls (sensitivity and specificity in the range of $85-90 \%) .{ }^{17}$ Typically, temporal and parietal hypometabolism and hypoperfusion are observed in $\mathrm{AD}$ patients relative to controls. However, because the added value of PET and SPECT over clinical diagnosis and structural imaging is not always clear, these investigations are not considered essential in the initial diagnostic work-up of dementia. ${ }^{2} \mathrm{~A}$ potential application is the early diagnosis of FTD, where a reduction in frontotemporal blood flow may be shown, even in the absence of evidence for frontotemporal atrophy.

An exciting novel application of PET is the in-vivo imaging of amyloid. The amyloid $\beta$ protein is considered essential to the pathogenesis of $\mathrm{AD}$, as it is the main constituent of neuritic plaques-one of the neuropathological hallmarks of AD. A gradual cortical deposition of amyloid $\beta$ into plaques was described by Braak and Braak, who proposed that amyloid plaques were first deposited in the basal neocortex, and subsequently spread to all areas of the cortex. ${ }^{6}$ Up until now, a definite diagnosis of $\mathrm{AD}$ can only be made post mortem, because the neuropathological characteristics of the disorder-neuritic plaques and neurofibrillary tangles-can only be observed at necropsy. If it were possible to detect amyloid $\beta$ in vivo, this would add considerably to the potential for an early diagnosis of $\mathrm{AD}$.

After years of preclinical research, several PET tracers have been developed for this purpose, and the first results in patients with $\mathrm{AD}$ are encouraging. Two compounds-fluorine-18labelled FDDNP ([18F]FDDNP), ${ }^{18}$ and the Pittsburgh compound B (carbon-11-labelled PIB) ([11C]PIB) ${ }^{19}$-have been studied to date, but several others are currently being developed. [18F]FDDNP labels both neurofibrillary tangles and $\beta$-amyloid neuritic plaques. In contrast, [11C]PIB only labels amyloid deposits. Both tracers showed greater retention in $\mathrm{AD}$ patients when compared to controls in areas known to be affected by Alzheimer pathology. In addition, a negative correlation was observed between binding of FDDNP and dementia severity. PIB did not show any significant correlation between amyloid load and cognitive function of the $\mathrm{AD}$ patients. From neuropathological 

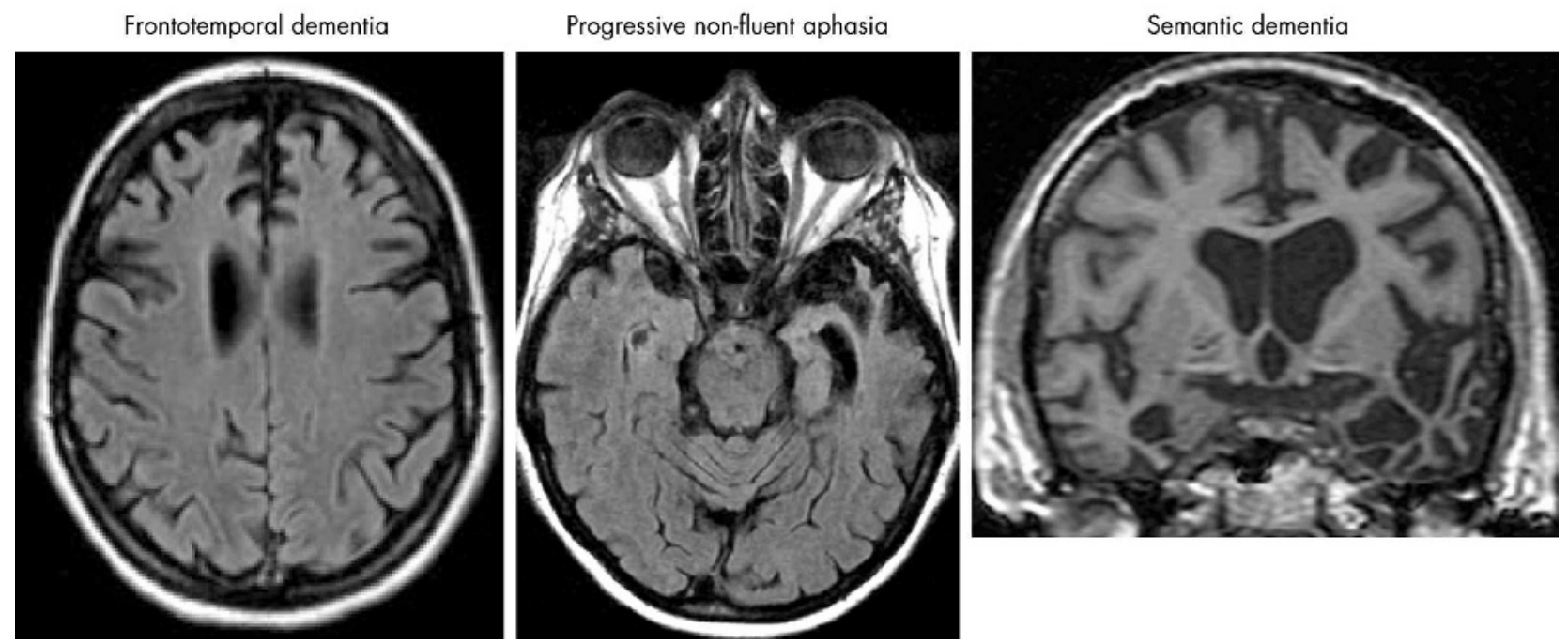

Figure 5 Axial MRI scans showing abnormalities in the spectrum of frontal temporal lobar degeneration. Frontotemporal dementia is associated with frontal and temporal atrophy. Progressive non-fluent aphasia typically presents with left sided perisylvian atrophy, whereas semantic dementia is characterised by left sided anterior temporal lobe atrophy.

studies, it is known that cognitive decline is associated with the progression of neurofibrillary tangles, rather than with the spread of neuritic plaques. This may explain the observation of an association between cognitive function and FDDNP (that seems to label both neurofibrillary tangles and amyloid containing neuritic plaques), but not between cognitive function and PIB (that seems to label amyloid deposition only).

For both tracers, many methodological issues still need to be resolved and the clinical value of in vivo amyloid imaging remains to be established. However, direct imaging of neuritic plaques, and hopefully also neurofibrillary tangles, is an exciting development that holds promise for the future. In vivo amyloid imaging may considerably add to our understanding of the underlying pathophysiological mechanisms of $\mathrm{AD}$ and could prove valuable as a surrogate marker in studies of anti-amyloid drugs.

\section{ELECTROENCEPHALOGRAPHY}

Generalised slowing of the background rhythm on electroencephalography (EEG) is a frequent finding in AD and DLB, and may be helpful in distinguishing such patients from those with depression. The described EEG changes are not specific for $\mathrm{AD}$, and can also be found in other diffuse neuroencephalopathies. In FTLD, the EEG may be normal until a far advanced disease stage. EEG seems unable to distinguish MCI patients from controls, nor to discriminate stable MCI patients from those who will convert to $\mathrm{AD}^{20}$ However, it has been suggested that EEG abnormalities in AD are suggestive of a faster progression of disease. ${ }^{21}$

The EEG may be a useful adjunct when there is diagnostic doubt. An abnormal EEG substantially raises the a priori chance of $\mathrm{AD}$, whereas a normal EEG has relatively little meaning. The observation of periodic sharp wave complexes is specific for Creutzfeld-Jakob disease (CJD), particularly the sporadic form. The sensitivity and specificity associated with the presence of these findings were $66 \%$ and $74 \%$, respectively. ${ }^{22}$ Epilepsy is a relatively rare cause of memory deficits. Transient epileptic amnesia caused by focal temporal lobe seizure activity can masquerade as AD. Even in patients that have no outward clinical manifestations of epilepsy, epileptiform abnormalities on EEG may have diagnostic importance.

\section{GENETIC TESTING}

Only a small proportion of all individuals with dementia suffer from a familial form, caused by an autosomal dominant mutation. There are a few types of dementia that are uniquely autosomal dominantly inherited, such as Huntington's disease and cerebral autosomal dominant arteriopathy with subcortical infarcts and leucoencephalopathy (CADASIL). Genetic testing for these diseases in specialised centres results in high sensitivity and specificity. By contrast, the largest proportion of patients suffering from the most widespread forms of dementia, such as $\mathrm{AD}, \mathrm{VaD}$, DLB, and FTLD, are "sporadic". In a minority of these patients, these types of dementia occur as autosomal dominant these types of disorders, with similar clinical presentation, although often with an earlier age of onset. Mutations in several genes (including $\mathrm{A} \beta$ precursor protein, presenilin 1, and presenilin 2) have been shown to cause AD, but these account for less than $5 \%$ of all cases. Tau mutations are found in some cases of familial FTLD and mutations in the prion protein gene are observed in familial CJD. The yield from mutation screening in unselected populations is exceedingly low. Genetic testing is thus not recommended unless a patient presents with an appropriate phenotype and a significant family history.

Genetic risk factors may also play a role in the so-called sporadic cases of dementia. In $\mathrm{AD}$, the apoliprotein $\mathrm{E}$ (APOE) $\epsilon 4$ polymorphism operates as a genetic risk modifier for $\mathrm{AD} .^{23}$ The presence of the APOE $\in 4$ isoform slightly increased the positive predictive value of the $\mathrm{AD}$ diagnosis. ${ }^{24}$ Relative to the neuropathological diagnosis of $\mathrm{AD}$, the sensitivity of the clinical diagnosis was $92 \%$, whereas the sensitivity of having at least one APOE $€ 4$ was only $65 \%$. However, in patients with clinical diagnoses of $\mathrm{AD}$, the addition of APOE testing increased the positive predictive value of a neuropathological diagnosis of $\mathrm{AD}$ from $90 \%$ to $94 \%$ if at least one APOE $€ 4$ allele was present. In patients with a clinical diagnosis of non-AD, the absence of an APOE $\in 4$ allele increased the 


\section{Abbreviations}

AD: Alzheimer's disease

APOE: apoliprotein E

- CADASIL: cerebral autosomal dominant arteriopathy with subcortical infarcts and leucoencephalopathy

- CJD: Creutzfeld-Jakob disease

- CT: computed tomography

- CSF: cerebrospinal fluid

DLB: dementia with Lewy bodies

- DSM IV: Diagnostic and statistical manual of mental disorders, 4th ed

EEG: electroencephalography

ELISA: enzyme linked immunosorbent assay

FDG: [18F]fluorodeoxyglucose

FTD: frontotemporal dementia

- FTL: frontotemporal lobar degeneration

- MCI: mild cognitive impairment

MMSE: mini mental state examination

MRI: magnetic resonance imaging

- NINDS-AIREN: National Institute of Neurological Disorders and Stroke-Association Internationale pour la Recherche et l'Enseignement en Neurosciences

- PET: positron emission tomography

- Ptau: phosphorylated tau

SPECT: single photon emission computed tomography

VaD: vascular dementia

negative predictive value from $64 \%$ to $72 \%$. Because of the relatively limited gain in diagnostic accuracy, routine use of APOE genotyping is currently not recommended in the diagnostic evaluation of dementia. ${ }^{2}$

\section{CEREBROSPINAL FLUID}

The blood-brain barrier restricts the flow of proteins to and from the brain, rendering biomarkers based on blood samples relatively unfit as measures of neurodegenerative disease. In contrast, cerebrospinal fluid (CSF) is in direct contact with the brain. CSF provides a "window to the brain" as biochemical changes resulting from pathological processes of the brain are reflected in it. Senile plaques and neurofibrillary tangles are the neuropathological hallmarks of AD. Neuritic plaques are caused by extracellular aggregation of $\beta$ amyloid $(A \beta)$ protein, and neurofibrillary tangles result from hyperphosphorylation of the protein tau. CSF 42 amino acid variant of $A \beta$ (A $\beta 42)$, total tau, and different epitopes of phospho-tau (ptau) are the most frequently used biomarkers of AD. For confirming the diagnosis of sporadic CJD, the 14-3-3 protein is useful.

\section{A $\beta 42$}

In patients with $\mathrm{AD}$, a decrease in CSF concentration of $\mathrm{A} \beta 42$ to about $50 \%$ of that in control subjects has been observed. The decrease in concentrations of CSF A $\beta 42$ in AD probably reflects deposition of the protein in plaques, as low concentrations of CSF A $\beta 42$ were found to be associated with high numbers of plaques in the neocortex and hippocampus. ${ }^{25}$ With specificity at $90 \%$, the mean sensitivity of CSF A $\beta 42$ is $86 \%$ when comparing $\mathrm{AD}$ with normal ageing. ${ }^{26}$ In the differential diagnosis between $\mathrm{AD}$ and other dementia disorders, CSF A $\beta 42$ is only moderately specific, as mildly reduced concentrations of CSF A $\beta 42$ have also been observed in other forms of dementia such as DLB, FTLD and VaD.
Tau

CSF tau concentrations are about three times higher in patients with $\mathrm{AD}$ than in normal ageing. Tau protein is located in the neuronal axons. The concentration of tau protein in the CSF probably reflects the intensity of neuronal degeneration in chronic neurodegenerative disorders. In a comparison between $\mathrm{AD}$ and controls, sensitivity seems to be somewhat lower than that of A 342 . With the specificity set at $90 \%$, the mean sensitivity of $\mathrm{AD}$ is $81 \%{ }^{26}$ Elevations of CSF tau levels are also observed in CJD and after acute stroke. Reports with respect to VaD and FTLD have been inconsistent.

\section{Phosphorylated tau}

The concentration of CSF phosphorylated tau protein (Ptau) may be more specific for $\mathrm{AD}$, as it specifically reflects the phosphorylation state of tau and, thus, the formation of tangles in $\mathrm{AD}$. In comparison with CSF A $\beta 42$ and tau, a considerably smaller number of studies have addressed sensitivity and specificity of Ptau. With a specificity of $92 \%$, the mean sensitivity of Ptau to discriminate AD from normal ageing is $80 \%$, although sensitivities vary widely among studies. ${ }^{26}$ It is important to notice that CSF Ptau may be more specific for $\mathrm{AD}$ than $\mathrm{A} \beta 42$ and total tau. High concentrations of CSF Ptau have only been observed in AD, whereas normal concentrations have been found in other dementia disorders such as DLB, VaD, and FTLD.

Studies of CSF biomarkers in mild dementia or MCI have suggested sensitivity figures comparable to those in later stages, indicating that CSF markers may reflect disease in a very early stage. Where CSF A $\beta 42$ and total tau differentiate dementia in general from normal ageing, CSF Ptau will increase the specificity of CSF biomarkers in the discrimination between $\mathrm{AD}$ and other dementias. The diagnostic yield may be improved by the simultaneous measurement of two biomarkers.

\section{4-3-3 protein}

Assessment of the 14-3-3 protein in the sporadic form of CJD has a sensitivity and specificity well above $90 \% .^{2}$ False positive results can be found in cerebral infarcts, encephalitis, tumours, and rapidly progressive AD. For the identification of CJD in cases with rapidly progressive dementia, assessment of the 14-3-3 protein yields the highest diagnostic accuracy.

The study of CSF biomarkers is a relatively new field and much work is still in progress. There is a lack of standardisation of the CSF assays. Concentrations of CSF markers have been found to vary among enzyme linked immunosorbent assay (ELISA) methods and between different centres using the same ELISA. Because of the variation in CSF values among studies, it has not been possible as yet to define generally applicable cut-off values for any of the CSF markers. This issue is currently addressed, as laboratories are compared on an international level. A second problem in the evaluation of the clinical usefulness of CSF biomarkers is the fact that almost all studies describe the results of CSF analysis in selected patient samples. There are virtually no studies that report on routine CSF analysis, which would be necessary to evaluate the clinical value of CSF analysis in an unbiased way. As yet, lumbar puncture is not recommended as a requirement for the diagnosis of dementia. However, results are promising and it is not unthinkable that, in the 
future, lumbar puncture with CSF analysis will be part of the routine diagnostic work-up in a memory clinic.

\section{DISCUSSION}

This overview has addressed the clinical diagnosis of dementia in general, and the potential for applying laboratory and imaging biomarkers in the quest for the specific neuropathology underlying dementia. Rather than giving a summary of diagnostic guidelines which can be found in other articles, ${ }^{2}$ we have attempted to lift the edge of the veil to the exciting new developments that may be incorporated in future guidelines.

The study of diagnostic markers for $\mathrm{AD}$ and other types of dementia is hampered by some methodological issues. The most important obstacle to the study of the diagnosis of $\mathrm{AD}$ is the lack of a gold standard. Almost all data come from clinical studies, with clinical diagnosis-based on careful application of clinical criteria-as the gold standard. This implies that the diagnostic performance of the marker under study can never exceed the diagnostic performance of the clinical diagnostic criteria that were used. This is especially a problem, as the quest for new biomarkers is instigated by the known insufficient sensitivity of clinical criteria in the earliest stages of neurodegenerative disease. If sensitivity and specificity are high, but below $100 \%$, does this mean that the new diagnostic marker is suboptimal, or does it reflect the flaws of the clinical criteria?

A second problem relates to the gradual accumulation of neuropathology and the insidious onset of clinical signs and symptoms. It is known that neuropathological changes eventually leading to dementia may start to accumulate as early as 20-30 years before a patient has clinical dementia. The question "where does ageing stop and dementia begin?" is closely followed by the question "how early should we diagnose?". Part of the control subjects in diagnostic studies of new biomarkers may develop dementia in later years. Thus, a finding of abnormal biomarkers in control subjects may either imply a false negative, or it may be a very early sign of imminent dementia. The suboptimal sensitivity and specificity figures of biomarkers may at least be partially due to poor selection of patient and control groups.

Another possible source of circular evidence originates in the multidisciplinary way the clinical diagnosis of dementia is made. When the new biomarker under study in fact contributes to the multidisciplinary diagnosis-or even when the multidisciplinary team is not completely blinded to the results of the new test-there is a risk of a self fulfilling prophecy. Selection bias occurs if a positive result on a new test contributes to the probability that a patient receives a specific diagnosis.

Finally, many markers of dementia show abnormalities in $\mathrm{AD}$ that are, however, not specific for $\mathrm{AD}$. This raises the question of whether the biomarker is not specific for the disease, or the disease itself is not specific? This question has received the most attention with respect to atrophy of the medial temporal lobe, although the issue equally affects other types of biomarkers. Atrophy of the medial temporal lobe is expected in $\mathrm{AD}$, as the neuropathology characteristic of $\mathrm{AD}$ has been shown to originate in this region. However, atrophy of the medial temporal lobe has also been reported in other types of dementia, such as VaD, DLB, and FTLD. The question is raised as to whether there is concomitant Alzheimer pathology in patients with these other types of dementia, or whether other types of neuropathology can also lead to atrophy in the medial temporal lobe. Currently, there is no definite answer for this question, although evidence accumulates that considerable overlap exists between different types of dementia. Evaluation of new biomarkers in large, unselected samples and clinical series with neuropathological confirmation of diagnosis is needed to shed more light on this discussion.

In conclusion, assessment of cognitive decline is essential to the clinical criteria of dementia, and biomarkers obtained using laboratory and imaging investigations can provide additional evidence for the presence of neuropathology. Although as yet there is no biomarker that can diagnose $\mathrm{AD}$ or any of the other major types of dementia with certainty, progress is being made and there are some promising new candidates for the detection of neuropathology.

(.................

Authors' affiliations

W M van der Flier, P Scheltens, Department of Neurology and Alzheimer Center, Vrije Universiteit Medical Center, Amsterdam, the Netherlands

\section{REFERENCES}

1 American Psychiatric Association. Diagnostic and statistical manual of mental disorders, 4th ed (DSM IV). Washington DC: APA, 1994.

2 Knopman DS, DeKosky ST, Cummings JL, et al. Practice parameter: diagnosis of dementia (an evidence-based review): report of the quality standards subcommittee of the American Academy of Neurology. Neurology 2001;56:1143-53.

3 Hejl A, Hogh P, Waldemar G. Potentially reversible conditions in 1000 consecutive memory clinic patients. J Neurol Neurosurg Psychiatry 2002;73:390-4.

4 Petersen RC, Smith GE, Waring SC, et al. Mild cognitive impairment: clinical characterization and outcome. Arch Neurol 1999;56:303-8.

5 Scheltens $P$, Fox N, Barkhof $F$, et al. Structural magnetic resonance imaging in the practical assessment of dementia: beyond exclusion. Lancet Neurol 2002;1:13-21.

6 Braak H, Braak E. Neuropathological staging of Alzheimer-related changes. Acta Neuropathol (Berl) $1991 ; 82: 239-59$

7 Gosche KM, Mortimer JA, Smith CD, et al. Hippocampal volume as an index of Alzheimer neuropathology: findings from the Nun study. Neurology 2002;58:1476-82.

8 Jack CRJ, Petersen RC, Xu YC, et al. Medial temporal atrophy on MRI in normal aging and very mild Alzheimer's disease. Neurology 1997:49:786-94.

9 Korf ES, Wahlund LO, Visser PJ, et al. Medial temporal lobe atrophy on MRI predicts dementia in patients with mild cognitive impairment. Neurology 2004;63:94-100

10 Fox NC, Warrington EK, Freeborough PA, et al. Presymptomatic hippocampal atrophy in Alzheimer's disease. A longitudinal MRI study. Brain 1996;119(Pt 6):2001-7.

11 Scheltens $\mathbf{P}$, Leys D, Barkhof F, et al. Atrophy of medial temporal lobes on MRI in "probable" Alzheimer's disease and normal ageing: diagnostic value and neuropsychological correlates. J Neurol Neurosurg Psychiatry 1992:55:967-72.

12 Neary D, Snowden JS, Gustafson L, et al. Frontotemporal lobar degeneration: a consensus on clinical diagnostic criteria. Neurology 1998;51:1546-54.

13 Román GC, Tatemichi TK, Erkinjuntti T, et al. Vascular dementia: diagnostic criteria for research studies. Report of the NINDS-AIREN International Workshop. Neurology 1993;43:250-60.

14 Van Straaten EC, Scheltens P, Knol DL, et al. Operational definitions for the NINDS-AIREN criteria for vascular dementia. An interobserver study. Stroke 2003;34:1907-12.

15 Snowdon DA, Greiner LH, Mortimer JA, et al. Brain infarction and the clinical expression of Alzheimer disease. The Nun study. JAMA 1997;277:813-17.

16 Medical Research Council CFAS Investigators. Pathological correlates of late-onset dementia in a multicentre, community-based population in England and Wales. Neuropathology group of the Medical Research Council cognitive function and ageing study (MRC CFAS). Lancet 2001;357:169-75.

17 Jagust W. Molecular neuroimaging in Alzheimer's disease. NeuroRx 2004; 1:206-12.

18 Shoghi-Jadid K, Small GW, Agdeppa ED, et al. Localization of neurofibrillary tangles and beta-amyloid plaques in the brains of living patients with Alzheimer disease. Am J Geriatr Psychiatry 2002;10:24-35. 
19 Klunk WE, Engler H, Nordberg A, et al. Imaging brain amyloid in Alzheimer's disease with Pittsburgh compound-B. Ann Neurol 2004;55:306-19.

20 Jelic V, Johansson SE, Almkvist O, et al. Quantitative electroencephalography in mild cognitive impairment: longitudinal changes and possible prediction of Alzheimer's disease. Neurobiol Aging 2000;21:533-40.

21 Claus $\mathrm{JJ}, \mathrm{Kwa} \mathrm{VI}$, Teunisse S, et al. Slowing on quantitative spectral EEG is a marker for rate of subsequent cognitive and functional decline in early Alzheimer disease. Alzheimer Dis Assoc Disord 1998;12:167-74.
23 Strittmatter WJ, Roses AD. Apolipoprotein E and Alzheimer disease. Proc Natl Acad Sci U S A 1995;92:4725-7.

24 Mayeux R, Saunders AM, Shea S, et al. Utility of the apolipoprotein E genotype in the diagnosis of Alzheimer's disease. Alzheimer's Disease Centers consortium on apolipoprotein E and Alzheimer's disease. NEngl J Med 1998:338:506-11.

25 Strozyk D, Blennow K, White LR, et al. CSF Abeta 42 levels correlate with amyloid-neuropathology in a population-based autopsy study. Neurology 2003:60:652-6.

26 Blennow K, Hampel H. CSF markers for incipient Alzheimer's disease. Lancet Neurol 2003;2:605-13. 